when blood enters one of them. Instances are recorded of fotuses born without brains and with only rudimentary spinal cords, and yet whose hearts have continued to act.

\section{CASE OF TRANSMISSION OF SECONDARY SYPHILIS: WITH REMARKS.}

By Thomas Skinner, M.D., Liverpool.

[Communicated to the Medical Institution of Liverpool, April 14,1864.] ON the 20th of September, 1861, I was consulted by a lady in reference to her first confinement. She was suffering from pruritus vulvæ, arising from a simple non-specific herpetic eruption on the vulva, which was cured in a week or two by diet and simple tonics, directed to improve the digestive organs. On the 4th of February following, she miscarried of a viable child, which, although seemingly healthy, died within a fortnight of its birth. The mother has had no return of the vaginal or vulvar irritation up to the present moment, and has enjoyed better health during all that time than has been her lot for some years before.

On the 3rd of July, 1862, she again conceived, went the full time of gestation without a single abnormal symptom, and was delivered of a rather puny-looking female child on the 10th of April, 1863. Within a week after birth, the child exhibited unmistakable signs of hereditary syphilis in an exaggerated form. On both hips, and around the anus and vulva, there was a florid red eruption, in rounded patches of various size and extent, each patch being raised above the level of the surrounding skin; and on the worst and most florid parts an ichorous discharge issued, which seemed to inoculate the sound skin, greatly extending thereby the diseased surface. Over the whole of the body a roseolar rash appeared, covered more or less with minutes scales, unaccompanied with itching. The whole of the mucous membrane of the mouth, lips, gums, fauces, and nostrils, became excoriated. The lips and gums, particularly the angles of the mouth, bled on the slightest touch; and they were always moist with a muco-serous exudation from the broken surfaces. Aphthous deposits were visible every where, but chietly on the tongue and buccal mucous membrane. The child began to emaciate rapidly; and the mother's milk disappeared, apparently from grief at seeing her infant in such a fightfully diseased condition; so that we were driven to the alternative of substituting a wet-nurse. A strong healthy wet-nurse was obtained; the child was put upon grey powder; the diseased mucous and cutaneous surfaces were dressed thrice daily with a weak solution of the acid nitrate of mercury; and in three weeks the child was free from any appearance of disease; but, as it was still weak and delicate, I sent it, with the nurse and father and mother, to the coast of North Wales, where it throve well, and remained quite well for a month, when a slight threatening of the eruption reappeared on the hips and vulva, with at the same time ozæna. A few more powders of chalk and mercury, and applying the lotion of the acid nitrate locally, again removed all trace of the disease within a week. The child has been weaned, and is now thriving better than ever, being both fat and plump.

So much for the disease in the child. But how did it come by it? As I have already stated, the mother never manifested a trace of syphilis, either locally or constitutionally, during either of her pregnancies; and from the 20 th of February, 1862, until the present date, she has never had any discharge, sore, or other local disturbance of the genito-urinary organs.
I at once suspected the husband; and I told him of my suspicions, when he candidly confessed that he had contracted the disease by a primary sore on the penis eight years ago; but, as he had undergone a thorough salivation on more than one occasion, he thought the poison had been eradicated from his system. On making further inquiries about his present state of health, I found that he was still the subject of constitutional syphilis. The form in which it now manifested itself was that of chronic syphilitic ulceration of the tongue, coming and going, not yielding to ordinary tonics. I examined the ulcers; and, judging from their characteristic ash-coloured surface, combined with the fact that they had come and gone for eight years independent of external causes, I have no doubt in my own mind that the husband was the cause of the disease in the child. He has since been cured to all appearance by iodide of iron, baths of nitro-muriatic acid, and an occasional mercurial purgative.

Having now accounted for the disease in the child, let us turn to the wet-nurse. The child had not been a week at the breast when, with one of its nails, it injured or broke the skin a little below the nipple. Every care was taken of this broken surface, by means of cleanliness, etc.; but I fear they were too late of adoption, and that some of the saliva or the secretion from the excoriated lips of the child inoculated the wound. The wound did not heal kindly more or less of a suppurative tendency showed itself. It healed with simple dressing within a week or ten days; and the woman went with the family to the coast, and remained out of my supervision for about four weeks. On her return, she was covered from head to foot with a roseolar rash, the same as I observed in the child a few days after its birth. On the back of the neck, and on other parts, the peculiar copper-coloured eruption of secondary syphilis was present; but I was very unwilling to believe it. Partly at my own request, and partly as it was the wish of the family, a second opinion was taken. The physician who met me was one who has had a very large experience of syphilis, and he at once pronounced it a genuine syphilitic eruption; in fact, he pronounced it so even before he had heard the history of the case; and I believe he was perfectly right. The wet-nurse was immediately put upon a course of mercurial purgatives, which removed all trace of the disease in about six weeks, without affecting the breast-milk otherwise than by increasing the quantity of the secretion. At this date, she remains perfectly free from any trace of the disease.

Remarks. I am fully aware that, so far as the profession as a body is concerned, we are far from being unanimous as regards the question of the possibility of the transmission of secondary or constitutional syphilis. My object in bringing this case before you is to strengthen our belief not only in the possibility, but in the certainty of such transmission as also that we may see how deeply we are interested in the subject in a legal sense, as regards the general management of such cases.

The case I consider quite equal to an experimentum crucis; and I feel perfectly confident that every avenue leading to deception or mistake has been strictly guarded. I can vouch for it that, during these two years, neither locally nor constitutionally has the mother ever manifested any appearance of a primary syphilitic sore or sores; and far less has she shown any symptom at all approaching to constitutional syphilis. I can also testify that, during the same period, she never was treated with any antisyphilitic agent. If you ask me how the mother escaped contagion, I answer that I know not. All that I can say is, that this case is not the only one 
on record where a child has been born, not only with hereditary syphilis, but with scarlatina, variola, rubeola, and other contagious diseases, and where the mother showed not the slightest trace of any of these affections during or after gestation. Mr. Victor De Méric, in his Lettsomian Lectures in 1858, states that, out of twenty-three cases of congenital or infantile syphilis, "he perceived that in thirteen the mothers who had given birth to them remained in perfect health." That the disease was syphilis in the child I make no doubt. My description of it; the effect of the mercurial treatment, local and constitutional; and the fact of the father having had syphilitic ulceration of the tongue for eight years,-are strongly confirmatory.

Lastly, of the wet-nurse. Before she was engaged, I examined her as I would any other wet nurse, and found her in every respect a very healthy woman, with the finest breast of milk that I ever saw. From motives of prudence, we did not institute a vaginal examination of the woman after the syphilitic eruption appeared ; but, on inquiring of the servants who washed her linen, and from actual examination of the linen, there was never found any trace of matter or discharge of any kind upon her thrown-off underclothing. What goes still further to prove this point, is the fact that the secondary symptoms showed themselves when she was in North Wales; an interval of four or five weeks having elapsed from the time that the child scratched the left breast.

\section{RETROSPECTIVE NOTES ON OUT- PATIENT PRAC'IICE.}

By C. M. Durrann, M.D., Phycician to the East Suffolk aid Tpswich Hospital.

$$
\text { [Continued from } p .88 \text {.] }
$$

6. Emphysema. We have had a few well marked examples of this disease in the past two years. Like asthma (although depending upon reiy different pathological causes), its symptoms are very capricious; and it is striking to see what liberties, in reference to diet and exposure, a patient may take in one locality, while, in another, the most vigilant care is futile in warding off an attack. As it is now very generally believed that the pulmonary lesion is produced by orer-forced expiratory, rather than by inspiratory effort, it behoves us to caution the labouring poor against an employment that will call for violent exertion while the breath is temporarily suspended, as in pushing or lifting heavy weights, etc.

Emphysema, whether vesicular or interlobular, is best relieved by strict attention to the general health. Flatulent dyspepsia will naturally much aggravate the attack of dyspncea; and, a rule, both in this complaint and in asthma, I have found the recommendation of Dr. Salter a very valuable one-viz., to debar the patient, as far as possible, from taking food after the middle of the day. The breakfast and midday meal may be substantial ; but the lightest allowance, both in quantity and quality, should be taken in the evening. As a rule, also, I believe that stimulants, in the shape of wine or malt liquor, are better avoided.

Although the general application of blisters is thought to be questionable, I have certainly seen much relief obtained from a blister applied to the epigastrium. It operates partly, I imagine, by relieving gastric congestion, but more especially by the direct influence which it exerts upon the semilunar ganglion and branches of the pneumogastric nerve.

As medicines, alkalies and chloric ether are beneficial, and sometimes cod-liver oil may be given with advantage. I have seen also very great relief to the dyspncea, both of emphysema and asthma, obtained by liquor arsenicalis, continued for some length of time.

Smoking stramonium and tobaceo may be of temporary service; as is also the inhalation of the fumes of paper prepared with a saturated solution of the nitrate of potash.

It will be often found, however, in private practice, that a carefully regulated diet, with warm clothing, and residence (if possible) in a dry atmosphere, will effect more in reference to the patient's comfort than we shall be able to afford him by medicinal treatment. The strict avoidance of constipation is, I think, a matter to be attended to in the treatment.

Cinculatory System.
1. Hyperamia. Under the denomination of congestion, we have a large number of cases noted as having applied as out-patients in the two years.

The symptoms at once point to the cause; viz., a more or less loaded state of the capillaries throughout the body, turgescence of the veins, and a dark purple appearance of the mucous membranes. With these, there is generally, also, a thickly coated tongue, and all the symptoms of congestive dyspepsia. Among the out-patient poor, the actual quantity of blood is probably increased as far as its watery constituent is concerned; while, among the more wealthy, indolent, and free-living, the quality rather than the quantity of the blood is changed, and this by increase both of the fibrine and also of the red globules.

The hard working "servant of all work" appears to me to be particularly prone to this hyperæmic condition of the circulation. In two cases, in this class of life, which were severe, I prescribed venesection with great advantage.

In general, a light diet without stimulants, free purgation by saline aperients, and the following mixture $(P h$. Lond.), will be all that is necessary.

13o Potassw bicarb. 3 iss ; potassce nitratis 3 ss ; tincturæ colchici $3 j$; spiritûs ammon. aromat. $j$ iss ; spiritûs æther. nitr. jij; misturæ camph. ad そviij. M. Sumat. partem sextam ter die.

2. Valvular nisease of the Heart. The most frequent form of cardiac disease that has obtained is lesion of the valves. Of these, the mitral have been most often affected; next, the mitral and aortic ; then, the aortic singly; and in one case, the semilunar pulmonary were alone implicated.

None of these cases were of recent date; and the majority could be traced to a rheumatic origin. In the treatment of mitral valve disease, the daily toil and struggle to live among some of the poor almost bopelessly negative our attempts to relieve.

Regulating the digestive function, allaying flatulence, and keeping the liver tolerably empty by the steady use of aperients, and giving iron in combination with alkalies, will, I think, be found the best general treatment of this affection.

In the treatment of the aortic lesion, which is far more hazardous to life than the former, I have found the following formula of Dr. Barlow certainly afford much relief, although it is difficult to say how it acts.

R Tincturæ hyoscyam., spiritûs ætheris nitr., i $3 \mathrm{ij}$; decocti senegr 3 ₹rs. M. Sumatur pars sexta ter die.

I have added bicarbonate of potash to the mixture if the digestive organs be faulty. If the patient be anæmic, steel is indicated; and in one case arsenic appeared to do much good. The dropsy, depending upon valvular lesion, obtains only in a limited extent among the out-patients; inasmuch as when 